\title{
Forecast for Full Life Cycle of Technical Renovation of Power Grid Based on Support Vector Machine
}

\author{
WU Zhuohang, YANG Jiye, CHEN Guolong, WANG Changchun, WU Hao, \\ GAO Qing
}

\author{
Liaoning Electric Power Co., Ltd. Liaoning Shenyang, China
}

Keywords: distribution network planning; life-cycle costs; cost control; economy

\begin{abstract}
Starting from the whole life cycle of the distribution network planning project, overall consideration should be given to the cost at different stages within the projection period distribution network planning project, and the time value of money. At the same time, consideration should also be given to the constraint conditions of the distribution network such as balance and others. Taking the total cost present value and its minimum of the multi-stage planning scheme of the distribution network structure as the objective function, this paper aims to establish the mathematical model of the multi-stage planning of the distribution network, so as to get the optimal planning scheme of the multi-stage planning of the distribution network which enjoys the coordination between reliability and economy.
\end{abstract}

\section{Introduction}

Distribution network is directly connected with the user, the power supply capacity and quality of the whole power system must be manifested through the distribution network. The reliability of the distribution system is the concentrated reflection of the structure and operating characteristics of the whole electricity system, its reliability assessment is an important part of the distribution network planning. When we apply the whole life cycle management concept to the distribution of the network planning, we need to give full consideration to the costs of investment, operations, and even abandoning at each stage, among them, the failure cost is directly reflected by the reliability and power loss of the planning scheme. In this case, choosing the scientific and rational power outage loss calculating method will be of great importance in calculating the cost of fault planning scheme.

\section{The target and research focus of the distribution network planning}

The planning and design of the distribution network can be divided into three parts: long-term planning, network planning, and construction, among them, long-term planning is the basic link of the electric power enterprise planning. In order to obtain as large as possible economic benefits, we should start from the viewpoint of the system's the long-term development. Planners should consider urban land price, urban construction layout, etc. Detailed analysis should be carried out on the city electricity supply. The optimal network connection form should be determined on the basis of the load prediction so as to determine the transient voltage, range and the optimizing investment cost.

Distribution network planning will determine the optimal system construction plan according to the basic condition of load forecasting results and existing networks during the planning. On the premise of meeting the load growth and supplying electricity safely and reliably, make the construction and operation cost of the distribution network be minimum. At various stages of planning, the reliability, safety and economy of the power supply should be adjusted timely, and the total cost within the system life cycle shall be kept as less as possible. Within the period of the power grid planning project, overall consideration should be given to all kinds of possible costs in the process of planning. We should maximize the economic benefits on the premise of ensuring the safe and stable operation and the power supply quality of the power grid. 


\section{Research focus of the distribution network planning}

Re-organize the voltage classes of the distribution network, and reduce the loss of distribution network. The changes of the voltage classes have a great influence on the economic efficiency of the distribution network investment and operation, it worth our studying. With more and more emphasis on the assessment research of the grid construction projects from grid company, the economic evaluation index system of the distribution network planning project and the evaluation methods have become hot topics. Building intelligent distribution network is the hot issue concerned by the grid company at present, and the application of distributed power supply is one of the core problems which will promote the constant going deeper of the planning study of the distribution network which contains the distributed generation. Now, the cry for distribution electricity price reform becomes increasingly stronger which make the electricity price regulation changes and the influence of electric market reform on the distribution network planning model become the research topic with forward-looking significance. Distribution network structure planning based on the total cost of life cycle. In the process of power distribution network planning, the planners need to improve the whole process of engineering cost management mode of China's traditional power construction project. Starting from the decision phase, we should give comprehensive consideration to the one-off construction cost and future operations, failure costs, and the best balance between different costs.

\section{Total life cycle cost}

The total life cycle cost management of the power grid planning projects is to apply the concept of whole life cycle into the electrical power system. Taking the costs of all the stages during the life circle of the planning project, and taking the optimum economic efficiency of the whole life cycle of planning project as the objective function, seek the best planning scheme which can satisfy the economic and social benefits at the same time. In the power grid planning project, implement the full life cycle management concept. On the premise of ensuring the safe and stable power supply, obtain the highest economic benefits with more reasonable cost, and achieve the maximization of the full life cycle overall benefits of the enterprise assets. Currently, the whole life-cycle theory has been widely used in the power system, and has achieved good social and economic benefits.

Although China's total life cycle cost technology research starts late, it develops rapidly. In the field of power system, East China Power Grid, Shanghai Electric Power and universities take the leading role in the technology research of the whole life cycle. From the point of research status at home and abroad, full life cycle technology has made certain achievements, though there are still some shortcomings and limitations. For example: as for single phase or products, it lacks of actual cases generally; the model is relatively simple. At the same time, the application of the whole life cycle technology in China is not so wide. Generally speaking, the full consideration is failed to give to the connection between the equipment and system. There is no concept of a whole. Therefore, whole life cycle technology enjoys forward looking significance on the application of power system internationally.

\section{Multi-stage optimizing planning of power grids based on the total life cycle cost}

Taking the total life cycle cost of the distribution network structure as the research object, give overall consideration to the total cost during the whole period from research and design to the end, and seek the planning scheme of the multi-stage power distribution network frame which can satisfy the constraint conditions, and enjoy reliable planning scheme and the optimal economic efficiency. Without getting involved in the substation site and construction planning cases of the transformer substation, according to the location of the power supply transformer substation, distribution network, load distribution and size of the distribution network power supply transformer substation, with reference to the existing network structure and parameters, taking the feeder as the research object and the total cost present value and the minimum cost of the whole life cycle multi-stage planning of the distribution network frame as the objective function, choose the line reasonably.

The mathematical model of the multi-stage planning of the distribution network frame: 
$P\left(A_{1}, \cdots, A_{N}, C\right)=\prod_{i=1}^{N} P\left(A_{i} \mid C\right) P(C)$

Constraints: $\left\{\begin{array}{l}\mathbf{V}_{H}=\mathbf{A}^{1} \cup \cdots \cup \mathbf{A}^{N_{L}} \\ \mathbf{A}^{l}=\left\{\mathbf{A}^{I^{1}}, \cdots, \mathbf{A}^{I_{L S}},\right\}\end{array}\right.$, the radial network operation mode.

\section{Key technologies of the total life cycle cost in the grid network planning}

It refers to establish corresponding model and solve it effectively with full consideration to the influence of all kinds of uncertain factors. Now, the uncertain factors modeling can achieve good results direct at one certain uncertain factor, while its influence on the other uncertain factors is limited. So we must speed up the research on the uncertainties modeling, and establish corresponding model for the various uncertain factors in the power system comprehensively accurately and solve it effectively.

It means to establish models of different rules and purposes and solve then effectively based on LCC technology. The purpose of the current power grid planning tends to have more diversity, such as power generation capacity adaptability, transmission congestion and grid income, etc. In order to meet the future planning of flexible grid, it is proposed to establish LCC models with different planning purposes, while finding the optimal solution to the model.

\section{The meaning of whole life cycle cost controlling}

Achieve cost optimization and resource saving. New ideas and new methods of the total life cycle cost controlling can guide the people to consciously, comprehensively start from the whole life cycle of the construction projects and give full consideration to the project construction cost, the operation and maintenance costs, so as to achieve the optimization and saving of the grid construction project cost. Examining the construction project cost controlling from the life cycle perspective can fully consider the initial costs and future costs of the construction projects, so as to achieve the minimum resource consumption within the life cycle of the grid construction project.

It will be helpful to speed up the "green" processes of the national construction industry. The "Eleventh Five-Year" Plan requires further promotion of green building and energy saving buildings which can let the whole society building energy consumption reach to the total target of $65 \%$ energy-saving. Analysis and evaluation on the all costs incurred by the throughout the life of the grid projects, and reducing the life cycle cost of green building is conducive to the promotion of green building, helpful to speed up the "green" process of the construction industry.

\section{Conclusion}

In the process of the long-term planning, we shall give dynamic consideration to the change of the load at different stages. Through reasonable planning, coordinating the relationship between the reliability and economy of the distribution network, we will strive to achieve the optimal economic efficiency while meeting the reliability of power supply which is also the focus of the current distribution network planning study. The concept of life cycle cost can make the power grid to achieve the optimum comprehensive benefit within the period of the whole life cycle, its model can ensure that the theory is applied in the power grid planning. Currently, the whole life cycle cost model still needs to be improved. Through the pilot study of the total life cycle cost in the power grid project, to build a more reasonable and effective optimal model of life cycle cost so as to promote the development of the power grid. 


\section{References}

[1]Guanqun Bao, Liang Mi, Yishuang Geng, Mingda Zhou, Kaveh Pahlavan, A video-based speed estimation technique for localizing the wireless capsule endoscope inside gastrointestinal tract, 2014 36th Annual International Conference of the IEEE Engineering in Medicine and Biology Society (EMBC), Aug. 2014

[2]Degui Zeng, Yishuang Geng, Content distribution mechanism in mobile P2P network, Journal of Networks, 9(5), 1229-1236, Jan. 2014

[3]Mingda Zhou, Guanqun Bao, Yishuang Geng, Bader Alkandari, Xiaoxi Li, Polyp detection and radius measurement in small intestine using video capsule endoscopy, 2014 7th International Conference on Biomedical Engineering and Informatics (BMEI), Oct. 2014

[4]Gan Yan, Yuxiang Lv, Qiyin Wang, Yishuang Geng, Routing algorithm based on delay rate in wireless cognitive radio network, Journal of Networks, 9(4), 948-955, Jan. 2014

[5]Guanqun Bao, Liang Mi, Yishuang Geng, Kaveh Pahlavan, A computer vision based speed estimation technique for localiz ing the wireless capsule endoscope inside small intestine, 36th Annual International Conference of the IEEE Engineering in Medicine and Biology Society (EMBC), Aug. 2014

[6]Xinchao Song, Yishuang Geng, Distributed community detection optimization algorithm for complex networks, Journal of Networks, 9(10), 2758-2765, Jan. 2014 